\title{
Compression of a pressurized spherical shell by a spherical or flat probe
}

\author{
Étienne Couturier ${ }^{1}$, Dominic Vella ${ }^{2}$, and Arezki Boudaoud ${ }^{3}$ \\ ${ }^{1}$ Laboratoire Matière et Systèmes Complexes, Université de Paris \\ CNRS UMR 7057, 10 Rue Alice Domont et Léonie Ducquet, 75205 \\ Paris Cedex 13, France \\ ${ }^{2}$ Mathematical Institute, University of Oxford, Woodstock Rd, \\ Oxford, OX2 6GG, UK \\ ${ }^{3}$ LadHyX, CNRS, Ecole polytechnique, IP Paris, 91128 Palaiseau \\ Cedex, France
}

February 15, 2022

\begin{abstract}
Measuring the mechanical properties of cells and tissues often involves indentation with a sphere, or compression between two plates. Different theoretical approaches have been developed to retrieve material parameters (e.g. elastic modulus) or state variables (e.g. pressure) from such experiments. Here, we extend previous theoretical work on indentation of a spherical pressurized shell by a point force to cover indentation by a spherical probe or a plate. We provide formulae that enable the modulus or pressure to be deduced from experimental results with realistic contact geometries, giving different results that are applicable depending on pressure level. We expect our results to be broadly useful when investigating biomechanics or mechanobiology of cells and tissues.
\end{abstract}

\section{Introduction}

In 1932, K. Cole Col32 introduced the mechanical compression of a sea urchin egg cell between two plates as a way to probe cell mechanical properties. This technique is now widely applied to both living and non-living objects ranging from microcapsules BOO13 and cell nuclei Gos+20 to single animal Hir63 64, plant BBT00 or, yeast ZBT99] cells, as well as animal embryos [Dav+99] and multicellular spheroids $[$ Sti+13] to name a few examples.

As might be expected with such a range of applications, nuances and differences of protocol have arisen, particularly with regard to the type of 
loading that is used. Different types of loading have different advantages and disadvantages: parallel plate compression is generally used to probe cells globally, while atomic force microscopy (AFM) has been used to make more local measurements Alc+07, MBB11. In the first implementations of AFM, the cantilevers had relatively sharp pyramidal tips, which could damage cells. Spherical beads used as tips appear to be less invasive and to generate less damage than sharp tips; they also make it possible to modify the range of applied stress by changing the bead diameter Mah+00.

Despite differences in loading protocol, all experimental approaches are similar in that they yield data for the force as a function of plate/tip displacement (or vice versa). Interpreting such force-displacement curves is therefore key, yet, since these curves depend on sample geometry and structure, the deduction of material parameters involves the use of mechanical models. In several cases, the sample can be approximated as a spherical thin shell, which might correspond to the cortex of an animal cell, the cell wall of a plant cell, or a peripheral stiff cell layer. Moreover, this shell is often pressurized from inside, whether that pressure arises from cytoplasmic pressure in the case of a single cell or pressure from inner cells in a multicellular spheroid. Here we aim to establish a suitable model for the compression of a pressurized spherical shell by a rigid flat or spherical probe. The first related work in this regard concerned compression by a flat plate, but neglected the effect of bending stiffness within the shell [FP75]. This model was later corrected for the incompressibility of the cell fluid content LP80, though the effect of bending stiffness was still neglected.

Moduli estimated by compression between two plates may significantly differ from moduli estimated with an AFM tip due to the difference of scale at which cells are probed ([TND03], [Ste+11], [TSZ10] $)$. A model for a point-like probe indenting a pressurized shell was developed in Vel+12b. Vel+12a , yielding analytical formulae to retrieve both the inner pressure and Young's modulus of the shell. Nevertheless, a model applicable to probe sizes between a point (the idealized AFM tip) and a flat plate is still missing. Here we establish such a model: we use an efficient formulation for the nonlinear contact problem for a shallow shell without friction that was introduced in AP10 and provide an analytical solution of the linearized problem that is valid for small displacement. Beyond this we solve the nonlinear boundary value problem numerically.

\section{Formulation and numerical solutions}

We consider a rigid spherical probe of radius $R_{o}$ (the planar case is recovered by taking $R_{o}=\infty$ ) pressed against a spherical shell of radius $R$, thickness $h$, Poisson ratio $\nu$, and Young's modulus $E$, that is inflated by a pressure $P$. We assume axisymmetry and express all variables as functions of the curvilinear coordinate $s$ (along the meridional direction). The periphery of the contact region between the probe and the shell is defined by its curvilinear coordinate $s_{c}$. Following [AP10], we formulate the equations using the rotation $\Psi=-\mathrm{d} w / \mathrm{d} s, w$ being the displacement normal to the sphere, the meridional (the ss component of) membrane 
a
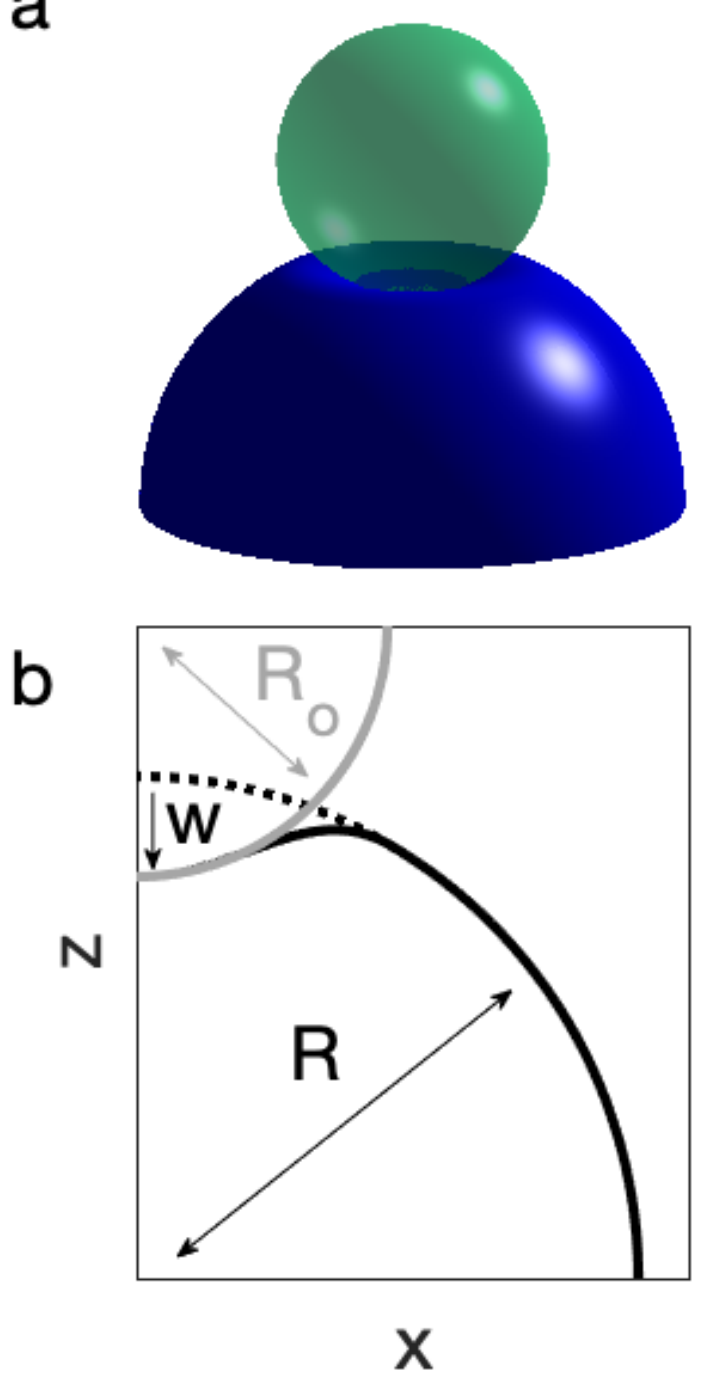

Figure 1: Geometry of the problem: a pressurized spherical shell in contact with a spherical probe. a. Three-dimensional schematic drawing showing the indenter (green, partially transparent sphere) indenting the shell (blue, deformed sphere). b. Cross-section and notations showing the pressurized sphere before indentation (dotted black curve), the pressurized sphere after indentation (continuous black curve) and the rigid spherical probe (grey curve). Here, $w$ is the normal displacement of the probe, while $R$ is the radius of the pressurized sphere and $R_{o}$ the radius of the rigid sphere (the indenter). 

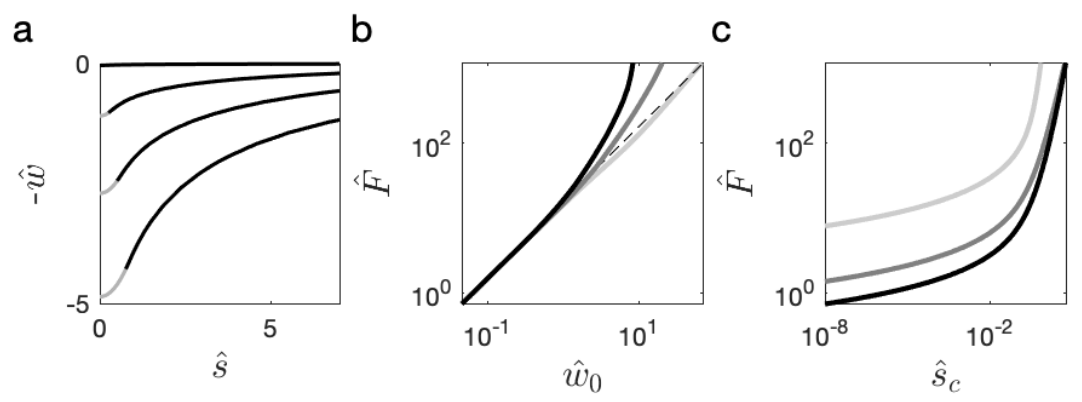

Figure 2: Numerical solutions for the contact of a pressurized spherical shell with a rigid spherical or flat probe. a. Dimensionless displacement $\hat{w}$ as a function of the dimensionless coordinate $\hat{s}$ for $\hat{p}=1$ and $R / R_{o}=1$. The grey (resp. black) part of the line correspond to the contact zone (resp. free zone). b. Dimensionless force as a function of the dimensionless displacement at the origin $\hat{w}_{0}$. The dashed line is a linear fit for small compressions $\hat{w} \lesssim 1$. c. Dimensionless force as a function of the dimensionless coordinate, $\hat{s}_{c}$, at which contact between the shell and the probe is lost. In (b) and (c), the dimensionless pressure $\hat{p}=8$; black, dark gray, and gray lines show results for $R / R_{o}=0$ (a plane), $R / R_{o}=1$, and $R / R_{o}=10$, respectively.

stress $\Sigma$, and the total reaction force integrated up to a disk of radius $s$, denoted $\phi(s)$ (see Figure 1 $1 \mathrm{p}$ ). The total contact force is $F=\phi\left(s_{c}\right)$. The governing equations are given by the compatibility equation AP10:

$$
\frac{3 s \Sigma^{\prime}+s^{2} \Sigma^{\prime \prime}}{E h}+s \frac{\Psi}{R}+\frac{\Psi^{2}}{2}=0,
$$

and by the vertical force balance

$$
s \Sigma\left(\frac{s}{R}+\Psi\right)-\frac{E h^{3}}{12\left(1-\nu^{2}\right)}\left(\frac{d\left(s \Psi^{\prime}\right)}{d s}+\frac{\Psi}{s}\right)=p \frac{s^{2}}{2}-\frac{\phi}{2 \pi} .
$$

These equations are complemented by the boundary conditions at the edge of the contact region, $s=s_{c}$, which require continuity of rotation, curvature and stress at the edge of the contact set, i.e.

$$
\begin{aligned}
\Psi\left(s_{c}\right) & =-s_{c}\left(\frac{1}{R}+\frac{1}{R_{o}}\right), \Psi^{\prime}\left(s_{c}\right)=-\left(\frac{1}{R}+\frac{1}{R_{o}}\right), \\
\Sigma^{\prime}\left(s_{c}\right) & =\frac{E h \sqrt{\epsilon} s_{c}}{8 R}\left(1-\left(\frac{R}{R_{o}}\right)^{2}\right),
\end{aligned}
$$

and boundary conditions at infinity, which require that the membrane stress returns to the isotropic value pre-compression (given by Laplace's equation) and that there is no rotation, i.e.

$$
\Sigma(\infty)=\frac{p R}{2}, \Psi(\infty)=0
$$


The hypothesis behind the kinematics are that strains are small but rotations may be moderate, i.e. that the typical rotation angle is much smaller than unity, but much larger than the membrane strain; these are expressed in eqns (12.11b), (14.11) and (14.12) of [AP10], for example. (The resulting equations are not exact but are a truncation of the exact results with the elastic strain terms truncated following the linear term in displacement, while rotation terms retain terms quadratic in the rotation - the smallest order term that includes the rotation.) The resulting equations also assume that the shell is "shallow" and hence are valid while displacements are restricted to a zone of the shell that is well approximated by a parabola centered at the apex, i.e.

$$
s \ll R, \quad s \ll R_{o}, z^{\prime}(s) \approx \frac{-s}{R}
$$

(There is no restriction on the indenter size, provided that the contact zone satisfies these requirements.)

The equations are made dimensionless by introducing the small parameter $\epsilon=\left(B / Y R^{2}\right)^{1 / 2}$ in which $B=E h^{3} /\left[12\left(1-\nu^{2}\right)\right]$ is the bending stiffness and $Y=E h$ is the membrane stretching stiffness; hence $\epsilon=$ $h / R /\left[12\left(1-\nu^{2}\right)\right]^{1 / 2} \ll 1$ for a thin shell. The balance between the terms representing bending stresses and those representing curvature-induced in-plane stresses in (2) introduces the bending length $R \sqrt{\epsilon} \sim(h R)^{1 / 2}$ as a natural unit for the curvilinear coordinate $s$ Pog73] we also use the shell radius $R$ as a unit of vertical displacement $w$, and $E \epsilon R h$ as the unit of force. The dimensionless quantities are then $\hat{s}=s /(R \sqrt{\epsilon})$, $\hat{w}=w / R, \hat{\Psi}=\Psi / \sqrt{\epsilon}, \hat{\Sigma}=\Sigma /(E h \epsilon), \hat{p}=p R /(E \epsilon h), \hat{\phi}=\phi /(E h R \epsilon)$, $\hat{F}=F /(E h R \epsilon)$. The compatibility and vertical force balance equations then take the form

$$
\begin{gathered}
3 \hat{s} \hat{\Sigma}^{\prime}+\hat{s}^{2} \hat{\Sigma}^{\prime \prime}+\hat{s} \hat{\Psi}+\frac{\hat{\Psi}^{2}}{2}=0 \\
\hat{s} \hat{\Sigma}(\hat{s}+\hat{\Psi})-\frac{d\left(\hat{s} \hat{\Psi}^{\prime}\right)}{d \hat{s}}+\frac{\hat{\Psi}}{\hat{s}}=\hat{p} \frac{\hat{s}^{2}}{2}-\frac{\hat{\phi}}{2 \pi} .
\end{gathered}
$$

Continuity conditions at the periphery of the contact region are

$\hat{\Psi}\left(\hat{s}_{c}\right)=-\hat{s}_{c}\left(1+\frac{R}{R_{o}}\right), \hat{\Psi}^{\prime}\left(\hat{s}_{c}\right)=-\left(1+\frac{R}{R_{o}}\right), \hat{\Sigma}^{\prime}\left(\hat{s}_{c}\right)=\frac{\hat{s}_{c}}{8}\left(1-\left(\frac{R}{R_{o}}\right)^{2}\right)$,

and the boundary conditions at infinity become

$$
\hat{\Sigma}(\infty)=\frac{\hat{p}}{2}, \hat{\Psi}(\infty)=0
$$

The system 6 - 6 is a fourth-order system with $\hat{F}$ determined as a function of $\hat{s}_{c}$ or vice-versa. The only other parameters in the problem are the dimensionless pressure, $\hat{p}$, and the ratio of shell to probe radius, $R / R_{o}$.

The boundary value problem was solved using the "bvp5c" solver of Matlab which provides $\hat{F}$ as part of the solution; the boundary conditions at infinity were imposed by continuation by varying an end point CK71. (Figure 2 a). As for a shell indented by a point tip, there is a linear relationship between force and displacement at small displacement (Figure 

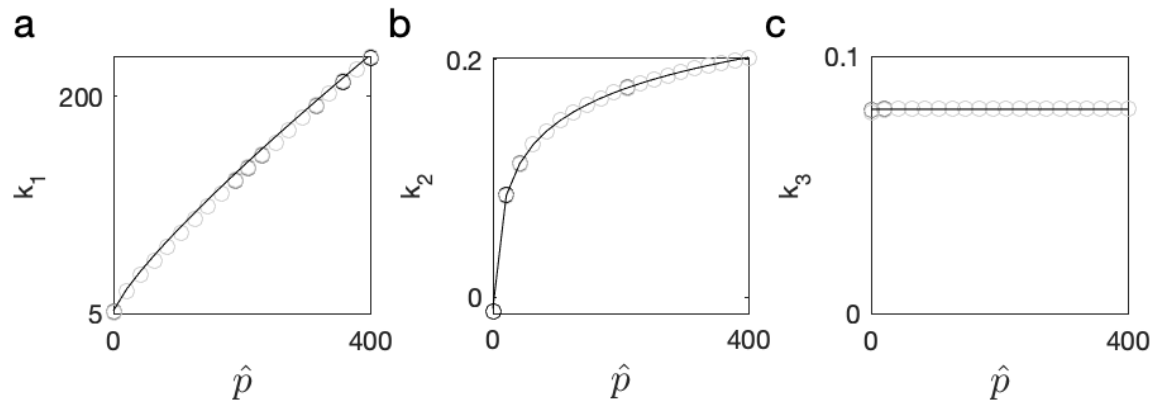

Figure 3: Comparison of analytical and numerical solutions at small displacement. Panels (a), (b), and (c) show the constants $k_{1}, k_{2}$, and $k_{3}$ that are involved in the equations relating size of contact region, probe displacement, and force, respectively, plotted as functions of the dimensionless pressure, $\hat{p}$. Circles represent the constants estimated from fits to numerical solutions and while curves show the predictions of the analytical solution of the linearized equations. Black, dark gray, and gray circles stand for $R / R_{o}=0$ (a plane), $R / R_{o}=1$, and $R / R_{o}=10$, respectively.

2 b). The numerical method works well at higher $\tau$ : the largest displacements that can be obtained numerically range from a few tenths of the thickness at $\tau=0$ to ten times the thickness at $\tau=1000$; the numerical accuracy closely follow the accuracy of the initial guess provided by the analytical solution. In particular, for contact with a plane at high $\tau$, and with large displacements, we find that the membrane limit is retrieved, i.e. the force scales as the area of the contact region multiplied by the pressure (data not shown). This reproduces the known membrane behavior of a pressurized membrane-shell, as found in the previous study by Feng \& Pangnan FP75.

\section{Small displacement behaviour}

For small displacement $(w \lesssim h)$, we expand equations (6)-(7) around the solution at zero force, $\hat{\psi}=0$ and $\hat{\Sigma}=\hat{p} / 2$. We linearize the equations in terms of $\hat{\Psi}$ and $\hat{\mu}=\hat{s}(\hat{\Sigma}-\hat{p} / 2)$ for $\hat{s}>\hat{s}_{c}$ :

$$
\begin{gathered}
\hat{\Psi}=-\frac{d}{d \hat{s}}\left[\frac{1}{\hat{s}} \frac{d}{d \hat{s}}(\hat{s} \hat{\mu})\right] \\
\hat{\mu} \hat{s}+\hat{s} \hat{\Psi} \frac{\hat{p}}{2}-\frac{d\left(\hat{s} \hat{\Psi}^{\prime}\right)}{d \hat{s}}+\frac{\hat{\Psi}}{\hat{s}}=\frac{\hat{F}}{2 \pi} .
\end{gathered}
$$

Substituting (10) into (11) yields a single fourth order equation for $\hat{\mu}$

$$
\hat{\mu}-\frac{\hat{p}}{2} \frac{d}{d \hat{s}}\left[\frac{1}{\hat{s}} \frac{d}{d \hat{s}}(\hat{s} \hat{\mu})\right]+\frac{d}{d \hat{s}}\left[\frac{1}{\hat{s}} \frac{d}{d \hat{s}}\left(\hat{s}\left(\frac{d}{d \hat{s}}\left[\frac{1}{\hat{s}} \frac{d}{d \hat{s}}(\hat{s} \hat{\mu})\right]\right)\right)\right]=\frac{\hat{F}}{2 \pi \hat{s}}
$$


The analytical solution is a sum of the particular solution $\hat{F} /(2 \pi \hat{s})$ and a solution of the homogeneous equation, i.e. a linear combination of the derivatives of $f_{+}(\hat{s})=K_{0}\left(\sqrt{\lambda_{+}} \hat{s}\right)$ and $f_{-}(\hat{s})=K_{0}\left(\sqrt{\lambda_{-}} \hat{s}\right), K_{0}$ being the zeroth order modified Bessel function of the second kind, and

$$
\lambda_{ \pm}=\hat{p} / 4 \pm \sqrt{(\hat{p} / 4)^{2}-1} .
$$

Accordingly, we may write (for $\hat{s}>\hat{s}_{c}$ )

$$
\hat{\mu}(\hat{s})=-\frac{a}{\lambda_{+}} f_{+}^{\prime}(\hat{s})-\frac{b}{\lambda_{-}} f_{-}^{\prime}(\hat{s})+\frac{\hat{F}}{2 \pi \hat{s}} .
$$

Substitution in 10 yields

$$
\hat{\Psi}(\hat{s})=a f_{+}^{\prime}(\hat{s})+b f_{-}^{\prime}(\hat{s})
$$

The boundary conditions at infinity are verified by the choice of $K_{0}(\cdot)$ and the constants $a$ and $b$ are obtained by imposing the boundary condition (8) for $\hat{\Psi}$ and $\hat{\Psi}^{\prime}$. We find that

$$
\begin{aligned}
& a=\left(1+\frac{R}{R_{o}}\right)\left[f_{-}^{\prime}\left(\hat{s}_{c}\right)-\hat{s}_{c} f_{-}^{\prime \prime}\left(\hat{s}_{c}\right)\right]\left[f_{+}^{\prime}\left(\hat{s}_{c}\right) f_{-}^{\prime \prime}\left(\hat{s}_{c}\right)-f_{-}^{\prime}\left(\hat{s}_{c}\right) f_{+}^{\prime \prime}\left(\hat{s}_{c}\right)\right]^{-1} \\
& b=\left(1+\frac{R}{R_{o}}\right)\left[f_{+}^{\prime}\left(\hat{s}_{c}\right)-\hat{s}_{c} f_{+}^{\prime \prime}\left(\hat{s}_{c}\right)\right]\left[f_{+}^{\prime}\left(\hat{s}_{c}\right) f_{-}^{\prime \prime}\left(\hat{s}_{c}\right)-f_{-}^{\prime}\left(\hat{s}_{c}\right) f_{+}^{\prime \prime}\left(\hat{s}_{c}\right)\right]^{-1}
\end{aligned}
$$

The boundary condition 8 for $\hat{\Sigma}^{\prime}$ yields a relation between $\hat{F}$ and $\hat{s_{c}}$ :

$$
\begin{aligned}
\hat{F} & =\left(1+\frac{R}{R_{o}}\right) \pi \frac{\left(f_{+}^{\prime}\left(\hat{s}_{c}\right)-\hat{s}_{c} f_{+}^{\prime \prime}\left(\hat{s}_{c}\right)\right)\left(f_{-}^{\prime}\left(\hat{s}_{c}\right)-\hat{s}_{c} f_{-}^{\prime \prime}\left(\hat{s}_{c}\right)\right) \sqrt{(\hat{p} / 4)^{2}-1}}{f_{+}^{\prime}\left(\hat{s}_{c}\right) f_{-}^{\prime \prime}\left(\hat{s}_{c}\right)-f_{-}^{\prime}\left(\hat{s}_{c}\right) f_{+}^{\prime \prime}\left(\hat{s}_{c}\right)} \\
& +\pi\left(1-\frac{R^{2}}{R_{o}^{2}}\right) \frac{\hat{s}_{c}^{4}}{8} .
\end{aligned}
$$

Finally we obtain the main physical quantities. From Eq. 14 the meridional membrane stress is

$$
\hat{\Sigma}_{s s}=\frac{\hat{p}}{2}-\frac{\hat{F}}{2 \pi \hat{s}^{2}}-\frac{a}{\hat{s} \lambda_{+}} f_{+}^{\prime}(\hat{s})-\frac{b}{\hat{s} \lambda_{-}} f_{-}^{\prime}(\hat{s}) .
$$

Following formula (14.20) of AP10, the dimensionless membrane circumferential stress is

$$
\hat{\Sigma}_{\theta \theta}=\frac{\hat{p}}{2}+\frac{\hat{F}}{2 \pi \hat{s}^{2}}-\frac{a}{\lambda_{+}} f_{+}^{\prime \prime}(\hat{s})-\frac{b}{\lambda_{-}} f_{-}^{\prime \prime}(\hat{s})
$$

The geometrical displacement is obtained by integrating (15):

$$
\hat{w}(\hat{s})=-a f_{+}(\hat{s})-b f_{-}(\hat{s}) .
$$

To obtain simple analytical formulae, we further consider the limit of a small contact region (relative to the bending length) i.e. $\hat{s}_{c} \ll 1-$ this is an approximation that holds for most of the range of simulations 
shown above. We also return to dimensional quantities, recalling that the dimensionless parameters

$$
\begin{aligned}
\epsilon & =\frac{h}{\left[12\left(1-\nu^{2}\right)\right]^{1 / 2} R} \\
\hat{p} & =\frac{p R}{\epsilon E h}
\end{aligned}
$$

with $\epsilon$ in particular used in the definition of the horizontal length scale.

The relation between the vertical displacement at the origin $w_{0}$ and $s_{c}$, the radius of the contact region, is given by

$$
w_{0}=R \epsilon \frac{1+R / R_{o}}{k_{1}\left[k_{2}-k_{3} \log \left(s_{c} /(R \sqrt{\epsilon})\right)\right]},
$$

where we introduce three constants

$$
\begin{gathered}
k_{1}=\frac{4 \pi \sqrt{(\hat{p} / 4)^{2}-1}}{\operatorname{arctanh}\left(\sqrt{1-(\hat{p} / 4)^{-2}}\right)}, \\
k_{2}=\frac{\hat{p}}{8 k_{1}}+\frac{\gamma-\log (2)}{4 \pi}, \\
k_{3}=1 / 4 \pi,
\end{gathered}
$$

and

where $\gamma$ is Euler's $\gamma$ constant. The first and third constants have the following limiting behaviours as a function of dimensionless pressure: $k_{1} \sim$ 8 and $k_{2} \sim \frac{\gamma-\log (2)}{4 \pi}$ for small $\hat{p}$, while $k_{1} \sim \pi \hat{p} / \log \hat{p}$ and $k_{2} \sim 1 /(8 \pi) \log \hat{p}$ for large $\hat{p}$. We manually got some point on the curve $(w$ vs $F /(2 \pi)$ ) of (AP10]) (Figure 14.15): the slope at the beginning is 1.39 which times $2 \pi$ gives 8.75 close from $k_{1}=8$. Note, in particular, that the expression 25 is well-defined and real for $\hat{p}<4$.

The relation between the force and $s_{c}$ is given by

$$
F=\epsilon^{2} E R h \frac{1+R / R_{o}}{k_{2}-k_{3} \log \left(s_{c} /(R \sqrt{\epsilon})\right)}
$$

Taking the ratio of 28 and 24 leads to a considerable simplification; at lowest order in $\hat{s}_{c}$, the force-displacement relationship is linear:

$$
F=\epsilon E h k_{1} w_{0} .
$$

Surprisingly, this equation does not involve the probe size, $R_{o}$. Indeed, the lowest order term (in $\hat{s}_{c}$ ) of $F / w$ that depends on $R / R_{o}$ is

$$
-\epsilon E h \pi\left(1-\frac{R}{R_{o}}\right) \frac{k_{1} k_{3} \log \left(s_{c} /(R / \sqrt{\epsilon})\right)\left(s_{c} /(R / \sqrt{\epsilon})\right)^{4}}{8}
$$

which remains negligible for small $\hat{s}_{c}$.

To compare these results with our numerical results, we fitted numerical solutions (Figure 3 a-c) to the functional forms 292824 , with $k_{1}$, $k_{2}$, and $k_{3}$ taken as free parameters, and we compared these values to the theoretical values Eqs. 25:27]. Consistent with our approximation, the match is good for small displacements when the pressure is high enough (Figure 3 a-c). The $w$ vs $F$ curve is independent of $R_{o} / R$ at lowest order, explaining that the dimensionless stiffness $k_{1}$ is identical to that obtained for a point indenter $\mathrm{Vel}+12 \mathrm{~b}$. 


\section{Conclusion}

We find that, at small compression, the pressurized spherical shell behaves as a linear spring with stiffness given by

$$
K=\epsilon E h \frac{4 \pi \sqrt{(\hat{p} / 4)^{2}-1}}{\operatorname{arctanh}\left(\sqrt{1-(\hat{p} / 4)^{-2}}\right)}
$$

(with $\epsilon$ and $\hat{p}$ defined by Eqs. 22 23) independently of the probe geometry. This result is potentially useful for the wide range of experimental protocols mentioned in the introduction. At low pressure, stiffness $K$ is mostly sensitive to modulus and at high pressure stiffness is mostly sensitive to pressure. Therefore, experiments in both normal medium and high-osmolarity media make it possible to deduce modulus and pressure (assuming Poisson's ratio has a standard value). If the monitoring of the contact area is possible, then the comparison of observed contact radius with theoretical radius at a given force $F$

$$
s_{c}=\sqrt{\epsilon} R \exp \left[4 \pi\left(k_{2}-\epsilon^{2} E R h\left(1+R / R_{o}\right) / F\right)\right]
$$

(with $\epsilon$ and $\hat{p}$ defined by Eqs. 22,23 and $k_{3}$ defined by Eq. 27) provides a simple test of whether it is appropriate to model the sample as a pressurized shell.

There are an increasing number of studies on mechanoperception and mechanotransduction in living systems - see reviews about plants [CMH21], animals VM17, ML16], fungi DM15], or bacteria [SJ11]. These studies call for theoretical descriptions of how external probes affect force distributions in cells and tissues. Indeed, the simplest experiment on invasive growth by plant roots or fungal hyphae involves putting the growing body in front of a plane and measuring the force upon impact $($ Biz+16], Mon01]). The deformation at the tip triggers a growth response: a quantitative study of this response first requires a precise description of tip deformation at contact, for which our study provides a first basis.

\section{$5 \quad$ Funding statement}

Etienne Couturier is funded by ANR AnAdSPi (ANR-20-CE30-0005). Arezki Boudaoud is funded by ANR-17-CE20-0023-02 WALLMIME, ANR20-CE13-0022-03 HydroField, ANR-20-CE13-0003-02 CellWallSense.

\section{Author contribution statement}

EC, DV and AB designed the research. EC perfomed the research. EC, $\mathrm{DV}$ and $\mathrm{AB}$ wrote the article. 


\section{References}

[Col32] Kenneth S Cole. "Surface forces of the Arbacia egg". In: Journal of Cellular and Comparative Physiology 1.1 (1932), pp. 1-9.

[Hir63] Y Hiramoto. "Mechanical properties of sea urchin eggs: I. Surface force and elastic modulus of the cell membrane". In: Experimental cell research 32.1 (1963), pp. 5975 .

[64] mitsuki yoneda mitsuki. "Tension at the surface of seaurchin egg: A critical examination of Cole's experiment". In: Journal of Experimental Biology 41.4 (1964), pp. 893906.

[CK71] Tuncer Cebeci and Herbert B Keller. "Shooting and parallel shooting methods for solving the Falkner-Skan boundary-layer equation". In: Journal of Computational Physics 7.2 (1971), pp. 289-300.

[Pog73] A. V. Pogorelov. Extrinsic geometry of convex surfaces. Vol. 35. American Mathematical Soc., 1973.

[FP75] William W. Feng and Huang Pangnan. "On the general contact problem of an inflated nonlinear plane membrane". In: International Journal of Solids and Structures 11.4 (1975), pp. 437-448. ISSN: 0020-7683. DOI: 10.1016/0020-7683(75)90079-7

[LP80] TJ Lardner and P Pujara. "Compression of spherical cells". In: Mechanics today. Elsevier, 1980, pp. 161-176.

[Dav+99] LA Davidson et al. "Measurements of mechanical properties of the blastula wall reveal which hypothesized mechanisms of primary invagination are physically plausible in the sea urchinstrongylocentrotus purpuratus". In: Developmental biology 209.2 (1999), pp. 221-238.

[ZBT99] Z Zhang, JM Blewett, and CR Thomas. "Modelling the effect of osmolality on the bursting strength of yeast cells". In: Journal of biotechnology 71.1-3 (1999), pp. 1724 .

[BBT00] Jenny Blewett, Kathleen Burrows, and Colin Thomas. "A micromanipulation method to measure the mechanical properties of single tomato suspension cells". In: Biotechnology letters 22.23 (2000), pp. 1877-1883.

[Mah+00] RE Mahaffy et al. "Scanning probe-based frequencydependent microrheology of polymer gels and biological cells". In: Physical review letters 85.4 (2000), p. 880. 
[Mon01] Nicholas P Money. "Biomechanics of invasive hyphal growth". In: Biology of the Fungal Cell. Springer, 2001, pp. $3-17$.

[TND03] Ahmed Touhami, Bernard Nysten, and Yves F Dufrêne. "Nanoscale mapping of the elasticity of microbial cells by atomic force microscopy". In: Langmuir 19.11 (2003), pp. 4539-4543.

[AP10] Basile Audoly and Yves Pomeau. Elasticity and geometry: from hair curls to the non-linear response of shells. Oxford University Press, 2010.

[TSZ10] Colin R Thomas, John D Stenson, and Zhibing Zhang. "Measuring the mechanical properties of single microbial cells". In: High Resolution Microbial Single Cell Analytics. Springer, 2010, pp. 83-98.

[Ste+11] John D Stenson et al. "Determining the mechanical properties of yeast cell walls". In: Biotechnology progress 27.2 (2011), pp. 505-512.

[SJ11] Sean X Sun and Hongyuan Jiang. "Physics of bacterial morphogenesis". eng. In: Microbiology and molecular biology reviews : MMBR 75.4 (Dec. 2011), pp. 543-565. DOI: $10.1128 / \mathrm{mmbr} .00006-11$

[Vel+12a] Dominic Vella et al. "Indentation of ellipsoidal and cylindrical elastic shells." English. In: Physical Review Letters 109.14 (Oct. 2012), p. 144302. DOI: 10.1103/physrevlett. 109.144302.

[Vel $+12 \mathrm{~b}]$ Dominic Vella et al. "The indentation of pressurized elastic shells: from polymeric capsules to yeast cells". In: Journal of the Royal Society Interface 9.68 (2012), pp. $448-455$.

[BOO13] Kiyoshi Bando, Kenkichi Ohba, and Yuta Oiso. "Deformation analysis of microcapsules compressed by two rigid parallel plates". In: Journal of biorheology 27.1-2 (2013), pp. 18-25.

[Sti+13] Tomita Vasilica Stirbat et al. "Multicellular aggregates: a model system for tissue rheology". In: The European Physical Journal E 36.8 (2013), p. 84. ISSN: 1292-8941. DOI: $10.1140 /$ epje/i2013-13084-1.

[DM15] Valeria Davì and Nicolas Minc. "Mechanics and morphogenesis of fission yeast cells". ENG. In: Current opinion in microbiology 28 (Aug. 2015), pp. 36-45. DOI: 10 . 1016/j.mib.2015.07.010. 
$[\mathrm{Biz}+16] \quad$ François Bizet et al. "3D deformation field in growing plant roots reveals both mechanical and biological responses to axial mechanical forces". In: Journal of Experimental Botany 67.19 (2016), pp. 5605-5614.

[ML16] Qiyan Mao and Thomas Lecuit. "Mechanochemical Interplay Drives Polarization in Cellular and Developmental Systems." English. In: Tissue Remodeling And Epithelial Morphogenesis 116 (2016), pp. 633-657. DOI: 10 . $1016 /$ bs.ctdb.2015.11.039

[VM17] Kyle H Vining and David J Mooney. "Mechanical forces direct stem cell behaviour in development and regeneration." English. In: Nature Reviews Molecular Cell Biology (Nov. 2017). DOI: $10.1038 / \mathrm{nrm} .2017 .108$.

[Gos+20] Rituparna Goswami et al. "Mechanical Shielding in Plant Nuclei." English. In: Current biology : CB 30.11 (June 2020), 2013-2025.e3. DOI: $10.1016 / \mathrm{j}$. cub. 2020.03. 059 .

[CMH21] Jennette M Codjoe, Kari Miller, and Elizabeth S Haswell. "PLANT CELL MECHANOBIOLOGY: GREATER THAN THE SUM OF ITS PARTS". In: The Plant Cell (2021), koab230-. ISSN: 1532-298X. DOI: 10.1093/plcell/ koab230.

$[$ Alc +07$]$ Jordi Alcaraz et al. "Bidirectional mechanobiology between cells and their local extracellular matrix probed by atomic force microscopy". English. In: Seminars in cell \& developmental biology (2017-07). DOI: 10.1016/ j.semcdb.2017.07.020. URL: http://www.sciencedirect. com/science/article/pii/S1084952117303282.

[MBB11] Pascale Milani, Siobhan A Braybrook, and Arezki Boudaoud. "Shrinking the hammer: micromechanical approaches to morphogenesis." English. In: Journal Of Experimental Botany 64.15 (2013-11), pp. 4651-4662. DOI: 10.1093/ jxb/ert169. 